Review

\title{
Nucleic acid $i$-motif structures in Analytical Chemistry
}

Joan Josep Alba, Anna Sadurní, Raimundo Gargallo*

Department of Analytical Chemistry, University of Barcelona, Martí i Franqués 1-11, E-08028 Barcelona, Spain

${ }^{*}$ Corresponding author

Phone: (+34) 934039116

Email: raimon_gargallo@ub.edu 


\begin{abstract}
Under the appropriate experimental conditions of $\mathrm{pH}$ and temperature, cytosine-rich segments in DNA or RNA sequences may produce a characteristic folded structure known as an i-motif. Besides its potential role in vivo, which is still under investigation, this structure has attracted increasing interest in other fields due to its sharp, fast and reversible $\mathrm{pH}$-driven conformational changes. This "on/off" switch at molecular level is being used in nanotechnology and analytical chemistry to develop nanomachines and sensors, respectively. This paper presents a review of the latest applications of this structure in the field of chemical analysis.
\end{abstract}

Keywords: Bioanalytical, Spectroscopy, Electrochemistry, i-motif, DNA 


\section{Introduction}

Besides the typical double helix structure described by Watson and Crick for DNA, nucleic acids may also form other structures depending on the sequences considered and the experimental conditions. For example, cytosine-rich DNA sequences may form a folded structure known as an i-motif, the stability of which is strongly dependent on $\mathrm{pH}$ and temperature, among other variables.

Until now, the $i$-motif has remained a relatively little known structure outside the field of nucleic acid research. However, it has recently gained the attention of an increasing number of research groups because it could also be an attractive drug for cancer treatment (Amato et al., 2014; Kendrick et al., 2014). i-motif structures have been observed in cytosine-rich sequences found at the end of telomeres (Leroy et al., 1994), in centromeres (Gallego et al., 1997), and near the promoter region of several oncogenes, such as bcl-2 (Kendrick et al., 2009; Khan et al., 2007), c-myc (Simonsson et al., 2000), and c-kit (Brazier et al., 2012; Bucek et al., 2010). It has also been postulated that several proteins may bind cytosine-rich sequences, despite the fact that it is not still clear whether the interaction takes place through the $i$-motif or through the unfolded strand (Fenn et al., 2007; Lacroix et al., 2000). Furthermore, the low $\mathrm{pH}$ of endosomes and the acid $\mathrm{pH}$ of the tumor microenvironment induced by the active metabolism of cancer cells have aroused interest in $\mathrm{pH}$ responsive systems for selective delivery (Webb et al., 2011).

Although the potential importance of $i$-motif structures in vivo is still under investigation, the applications of the sharp, fast and reversible $\mathrm{pH}$-driven conformational changes associated with this structure are currently being used in many other fields, such as Nanotechnology (Liu and Balasubramanian, 2003; Teller and Willner, 2010). These applications have been recently reviewed (Dong et al., 2014). Here, we review the proposed applications of this structure in the field of chemical analysis with the aim of providing a critical overview of $i$-motif applications that adds to the information given in two recently published reviews on fundamental aspects of the $i$-motif (Benabou et al., 2014; Day et al., 2014). First, we discuss the most basic chemical and biophysical characteristics of this structure. Then, we explore the most recent developments in $i$-motif structure detection, and continue with a description of the $i$-motif structure applications in chemical analysis. Finally, the perspectives of use of this structure in chemical analysis are commented, with special emphasis given to the limitations expected to drive the application of this structure. 


\subsection{The $i$-motif structure}

The $i$-motif structure is produced from the folding of cytosine-rich nucleic acid sequences. The core of the structure is formed by two parallel duplexes that are intercalated in an antiparallel manner (Figure 1a). It may be formed from the spatial arrangement of two or four independent strands (known as the bimolecular or tetramolecular i-motifs, respectively), or from the folding of a single strand (monomeric or intramolecular $i$-motif). Bimolecular and intramolecular $i$-motifs contain two and three loops, respectively, whereas tetramolecular $i$-motifs, which do not contain loop regions, show negatively-charged regions at both ends. The multimeric nature of $i$-motif folding is related to the number of cytosine bases and the length of loops (Mergny et al., 1995).

The building block of the $i$-motif structure is a base pair involving one neutral (deprotonated) cytosine and one positively-charged (protonated) cytosine at the N3 position. The resulting $C \cdot \mathrm{C}^{+}$base pair, which is stable because of the formation of three hydrogen bonds, allows the formation of the abovementioned parallel duplexes. Cytosine protonation in cytosine-rich sequences prone to form $i$-motif structures shows a different pattern to that observed in the free base, whose $\mathrm{pK}_{\mathrm{a}}$ value is around 4.5 (Figure $1 \mathrm{~b}$ ). At $\sim 25^{\circ} \mathrm{C}$, cytosine bases are deprotonated at $\mathrm{pH}$ values higher than approximately 6.5 , hindering the formation of C.C $\mathrm{C}^{+}$base pairs and consequently, that of the $i$-motif structure. In general, the stability of folded structures is inversely proportional to temperature. Thus, the formation of $i$-motif structures has been observed at $4^{\circ} \mathrm{C}$ even at slightly basic $\mathrm{pH}$ values (Zhou et al., 2012). At pH values lower than 6.5 and room temperature, the ensemble of cytosine bases is partially protonated and the nucleic acid folds into the $i$-motif structure. However, when the $\mathrm{pH}$ value falls below approximately 3 , all cytosine bases are protonated and formation of the C.C. $\mathrm{C}^{+}$base pair is hindered, dramatically reducing the stability of the $i$-motif structure.

The $\mathrm{pH}$-induced formation of folded structures such as the $i$-motif is accompanied by a strong cooperative effect inherent to their polymeric nature. Hence, the protonation of a neutral cytosine base leads to the formation of a C.C $\mathrm{C}^{+}$ base pair with a nearby neutral cytosine, which in turn favors the formation of additional $\mathrm{C} \cdot \mathrm{C}^{+}$base pairs by neighboring cytosines. As a result, the protonation of half of the cytosine ensemble in a cytosine-rich oligonucleotide takes place in a smaller $\mathrm{pH}$ range than in the case of an ensemble of free cytosines (Figure $1 \mathrm{~b}$ ). This effect is not observed in acid-base titrations of free cytosines because it is only present in polymers, where functional groups prone to protonate are close together. The magnitude of this cooperative effect is dramatically influenced by the 
number and arrangement of cytosine bases (Gurung et al., 2015; Nesterova et al., 2013). Recently, it has been shown that the presence of additional structural motifs such as internal or external hairpins may help to modulate the cooperative effects present in the formation of $i$-motif structures at neutral $\mathrm{pH}$ and room temperature (Nesterova and Nesterov, 2014), leading to the development of customized pH sensors.

The acid-base properties of $i$-motif structures are also dependent on the nature of additional chemical groups that may protonate or deprotonate in a $\mathrm{pH}$ range of approximately 3-7. Hence, the number and nature of bases at the loops strongly influence $\mathrm{pH}$-transition midpoints and the $\mathrm{pH}$ range of existence of mono- and bimolecular $i$-motif structures. It has been suggested that intramolecular $i$-motif structures showing long loop regions are thought to be more stable in the event of $\mathrm{pH}$ changes (Brooks et al., 2010). However, recent results have cast some doubt on the generality of this effect (Gurung et al., 2015; Reilly et al., 2015). Also, it has been observed that the presence of bulky adenine bases (the $\mathrm{pK}_{\mathrm{a}}$ of which is $\sim 3.5$ ) in the loops produces conformational changes involving disruption of the $i$ motif core (Lieblein et al., 2013). In contrast, mutation of TAA repeats for TTT in intramolecular i-motif structures formed by the cytosine-rich strand of the human telomere increases their stability (Fernandez et al., 2011). It has been suggested in a recent study that bases adjacent to the cytosine segments in loops 1 and 3 exert a strong influence on the stability of intramolecular $i$-motif structures (Fujii and Sugimoto, 2015). Thus, guanine or thymine bases in loops 1 and 3 positions adjacent to C-rich regions stabilize the structure due to inter-loop hydrogen bonding. Furthermore, additional $\mathrm{C} \cdot \mathrm{C}^{+}$base pairs located at loops 1 and 3 also increase the stability of the $i$-motif structure.

Kinetics of i-motif formation is a key point that must be taken into account when developing analytical methods based on its folding. Folding kinetics depends strongly on the monomeric or multimeric character of the resulting folded structure, as well as on other variables such as $\mathrm{pH}$ or temperature. Hence, cytosine-rich sequences fold faster in monomeric i-motif structures than in multimeric. In this later case, moreover, nucleic acid concentration plays an important role on kinetics. Most of the analytical methods based on the use of i-motif structures are based on monomeric i-motif structures, which fold in time scales of seconds (Liu and Balasubramanian, 2003). However, the formation of dimeric $\left(\mathrm{mC}_{2} \mathrm{TCACTC}\right)_{2}$ and tetrameric $\left(\mathrm{TC}_{3}\right)_{4}$ i-motifs takes place in hours (Canalia and Leroy, 2009; Canalia and Leroy, 2005). Moreover, dimeric and tetrameric structures may show more complex formation pathways than intramolecular structures (Leroy, 2009), affecting to the reproducibility of the analytical information. As a result, and as reviewed below, most of the described analytical methods make use of intramolecular structures, increasing in this way both, their responding speed and reproducibility. 
Not only oligodeoxynucleotides form i-motif structures, but also oligoribonucleotides. In general, however, RNA imotifs show lower stability than the corresponding DNA i-motifs (Lacroix et al., 1996). It has been proposed that the steric hindrance between $2^{\prime}$-hydroxyls in the narrow groove should be most responsible for the absence of stable imotifs structures (Collin and Gehring, 1998). Finally, nucleic acid sequences prone to form $i$-motif structures may also incorporate a plethora of chemical modifications in nitrogenous bases (Lannes et al., 2015), sugar moieties and/or backbone (Kumar et al., 2009; Perez-Rentero et al., 2015) that can affect the overall stability of the structure. At this point, it should be noted that $i$-motif structures consisting of natural nucleotides exist in a relative narrow $\mathrm{pH}$ range, from 3 to 7, approximately, at room temperature. Therefore, application of solution equilibria involving this structure for $\mathrm{pH}$ analysis at basic values is rather difficult. Finally, interaction with ligands such as porphyrins (Dexheimer et al., 2009) or carbon nanotubes (see below) may increase the stability of the $i$-motif structure. All these aspects will not be addressed here as they have been recently reviewed elsewhere (Amato et al., 2014; Benabou et al., 2014).

\section{Detection of $i$-motif structures}

The most usual methods for detecting the formation of $i$-motif structures in biophysical studies involve spectroscopic techniques such as circular dichroism (CD) or nuclear magnetic resonance (NMR). The characteristic CD signature of this structure shows two positive and negative signals centered at $\sim 286$ and $\sim 265 \mathrm{~nm}$, respectively, with the intensity of the first band being twice that of the second one. The NMR spectra of $i$-motif structures, on the other hand, show a characteristic group of signals at $\sim 15 \mathrm{ppm}$ due to imino protons. This is quite a selective signal because the Watson-Crick G.C and A.T base pairs show NMR signals between $\sim 12$ and $\sim 14$ ppm, whereas other non-canonical base pairs, such as T·T or G.T mismatches, show signals between $\sim 10$ and $\sim 12 \mathrm{ppm}$.

The formation of $i$-motif structures in gas-phase has also been detected using electrospray ionization mass spectrometry (ESI-MS). In a pioneer study, gas-phase conformations of the cytosine-rich sequence found at the human telomere $\left(5^{\prime}-\left(C_{3} A_{2} T\right)_{3} C_{3}-3^{\prime}\right)$ were studied using ion mobility spectrometry as a function of the charge state (Rosu et al., 2010). It was shown that the negative ions of the lowest charge states corresponded to the preserved $i$ motif structure. Recently, MS has been used to study the formation of tetrameric $i$-motif structures (Cao et al., 2015b). Here, the ion complexes detected in the gas-phase showed direct evidence of a molecule-by-molecule 
formation and dissociation pathway of the resulting tetrameric $i$-motif. This suggests that the formation of a trimolecular structure is the rate-limiting step in this mechanism.

Approaches are being developed to detect the formation of $i$-motif structures in the framework of Watson-Crick duplex DNA regions, most of which are related to molecular fluorescence spectroscopy. Lee et al. have proposed a system for discriminating human $i$-motif structures based on the different stacking interactions between non-polar fluorescent molecules and a planar base pair at the terminal and mid-loop positions of human telomeric $i$-motif sequences (Lee et al., 2012; Lee et al., 2009). The molecule (pyrene or fluorene) is covalently attached to a nucleotide intercalated into the cytosine-rich sequence. In the presence of cytosine bases in either C.C $C^{+}$or C.G base pairs, dye fluorescence was dramatically reduced because of the formation of exciplex states. The exciplex was probably formed by $\pi$-electron coupling or stacking between the dye molecules and nucleobases in close vicinity. This approach coupled with the time-resolved fluorescence technique was used to study the formation of intermediate states such as partially folded $i$-motif structures which could not be observed using CD. More recently, Xu et al. have described the use of thiazole orange (TO) (Figure 2), which can also form fluorescent exciplex species, to monitor the formation of $i$-motif structures (Xu et al., 2014). The spatial orientation of the loop region in the $i$ motif supplied a defined scaffold to form a fluorescent exciplex between dye molecules covalently bonded to the DNA and nucleobases at the loops. Upon excitation with visible light, the single TO molecule emitted orange exciplex fluorescence in the $i$-motif structure and green fluorescence as a TO monomer in duplex DNA. This large Stokes shift, high quantum yield, robust response to $\mathrm{pH}$ oscillation and little structural disturbance to the $i$-motif structure, together indicate a reliable method for detecting $i$-motif formation.

Another approach for detecting the formation of $i$-motif structures is based on enhancing the fluorescence of a free dye upon interaction with the folded nucleic acid. The dyes reported to date for this purpose include crystal violet and thioflavin. Crystal violet has been shown to be a relatively selective fluorescent probe for $i$-motif structures in the presence of complementary guanine-rich regions, which have the capacity to fold into another complex structure known as the G-quadruplex in the presence of several cations, such as $\mathrm{K}^{+}$(Ma et al., 2011). Interaction between the dye and $i$-motif was predicted to involve external end-stacking according to molecular modeling calculations. Meanwhile, the ligand thioflavin has been shown to interact with two $i$-motif-forming sequences found in the RET and retinoblastoma (Rb) genes (Lee et al., 2015a). A dramatic change occurred in the fluorescence emission spectra of the dye in presence of all of these sequences, upon structural transition from random coil to $i$ - 
motif structure. Interestingly, opposite patterns were observed for fluorescence. When $\mathrm{pH}$ changed from $\mathrm{pH} 8$ to $\mathrm{pH}$ 5, RET sequence emission decreased by around 6.3 times, while in the case of the Rb gene, emission was increased by around 10 times. These increases in the fluorescence intensity of the dye were explained in terms of the formation of hairpin structures (for the RET gene at $\mathrm{pH}$ 8) and an $i$-motif structure showing a long -AAAA- loop (for the $\mathrm{Rb}$ gene at $\mathrm{pH}$ 5). Lastly, the use of a polythiophene derivative (PMNT) has been reported for detecting $i$-motif formation (Ren et al., 2010). This method is based on the change in color of water-soluble polythiophene derivatives owing to conformational changes in their flexible conjugated backbones, which can be induced by interaction with appropriate nucleic acid structures. In particular, a change in the absorption properties of polythiophene upon interaction with the $i$-motif structure leads to a change in the color of the polymer solution, which is visible even to the naked eye. Unfortunately, the selectivity of this method was not reported. Concurrently, Wang et al. used another polythiophene derivative as a sensing probe (Wang et al., 2010). Two sensing configurations were designed: one used the polymer alone to detect the reversible conversion between $i$-motif and random-coiled state of a cytosine-rich single-strand DNA, while the other used the polymer and a complementary single-strand DNA to investigate reversible conversion of the Watson-Crick duplex to $i$-motif equilibrium. All the conversions showed color changes visible to the naked eye within a few minutes. In this case, the reported limit of detection (LOD) was $40 \mathrm{nM}$. A related approach makes use of the disappearance of the fluorescence present in free dyes upon interaction with the $i$-motif structure. For example, a study has been conducted of the interaction of homodimeric cyanine dyes with i-motif-forming sequences (Ruedas-Rama et al., 2014). These dyes, which are fluorescent in their free form, may form non-fluorescent $\mathrm{H}$-aggregates (stacked dyes) when interacting with $\mathrm{i}$-motif structures. It was found that the formation of these $\mathrm{H}$-aggregates was promoted when at least six consecutive cytidines were present.

A more complex approach involves the use of two intercalating dyes (thiazole orange and ethidium bromide) and a cationic conjugated polymer (PFEP, a polyphene derivative) (Li et al., 2011). The fluorescence resonance energy transfer (FRET) process was modulated by the $\mathrm{pH}$-driven conformational conversion of the $i$-motif structure formed by the human telomeric cytosine-rich sequence. The system could be switched back and forth by additions of $\mathrm{H}^{+}$and $\mathrm{OH}^{-}$, and the FRET signal was used for label-free detection of conformational conversion of the $i$-motif structure. Another fluorescence-based technique, fluorescence anisotropy, has been used to detect the formation of $i$-motif structures. This consisted of measuring emission depolarization when the fluorophore was excited with polarized light. The measured anisotropy value was sensitive to the volume and structural changes of the molecule studied. In 
a recent work, this technique has been used to study the influence of buffer composition, cytosine segment length and the presence of the guanine-rich complementary DNA strand in the conformational equilibria of the $i$-motif structure (Huang et al., 2015a). Two different fluorophores, 6-carboxy-x-rhodamine (ROX) and 5-

carboxytetramethylrhodamine (TAMRA), both having positively charged centers that enabled a strong electrostatic interaction with the negatively charged DNA backbone, were evaluated. In both cases, the original weak fluorescence signal from the cytosine-rich strand labeled in its $5^{\prime}$-end with either ROX or TAMRA increased dramatically upon addition of acid. This fact was explained because of the higher value of the rotational correlation time attributed to the folded i-motif structure.

Lastly, a non-spectroscopic technique, size-exclusion chromatography (SEC), has been used to study complex DNA structures, including $i$-motifs (Largy and Mergny, 2014). SEC proved to be a simple and powerful tool to assess the secondary structure formed by oligonucleotides, and extensive calibration and validation was performed of the use of SEC to detect the presence of different species displaying various structures and/or molecularity. The study also described simple metrics that facilitated the use of SEC without the need for time-consuming calibration.

\section{Applications in chemical analysis}

\subsection{Spectroscopy-based pH analysis}

The stability of the $i$-motif structure is strongly $\mathrm{pH}$-dependent. Therefore, the most straightforward analytical application of this structure is evidently $\mathrm{pH}$ analysis. Several methods that leverage this behavior are under development, based on either spectroscopic or electrochemical analysis, both in vitro and in vivo. In the previous section, we reviewed $i$-motif structure detection by means of spectroscopic methods. Below, we review spectroscopic methods for $\mathrm{pH}$ measurement that are based on $\mathrm{pH}$-induced conformational changes in this structure.

Several colorimetric methods are based on the $\mathrm{pH}$-controlled aggregation of gold nanoparticles (AuNPs) because these sensors offer high sensitivity and ease of miniaturization. When dissolved in water, AuNPs show an intense red color due to plasmon coupling for particles measuring less than $100 \mathrm{~nm}$, or blue/purple for the larger particles resulting from aggregation. This process may be facilitated using the molecular recognition properties of DNA oligonucleotides, where these are chemically linked to the surface of AuNPs by gold-thiol chemistry (Loweth et al., 1999). AuNP aggregation can also be triggered by environmental changes, such as $\mathrm{pH}$ variations, when working with 
cytosine-rich sequences with the capacity to fold into $i$-motif structures. One of the first applications of AuNPs in pH analysis comprised two different sets of DNA-AuNP conjugates, NP1 and NP2 (Sharma et al., 2007). NP1 consisted of AuNPs conjugated with a cytosine-rich DNA strand (30 nucleotides long) containing four segments of cytosine bases, while the NP2 consisted of AuNPs conjugated with a DNA strand (27 nucleotides long) complementary to NP1 but with 3 C.T mismatches (Figure 3). At neutral pH, the cytosine-rich and guanine-rich strands formed the Watson-Crick duplex, favoring the formation of AuNP aggregates, colored purple. In contrast, at $\mathrm{pH}^{\sim 5}$, the $i$-motif was formed, preventing the formation of AuNP aggregates and leading to a clear red color. The main advantage of this method is that $\mathrm{pH}$ changes can be detected by the naked eye. However, it should be noted that the color changes took place completely in $\sim 30$ minutes. Interestingly, it was only upon addition of $\mathrm{NaCl}(>150 \mathrm{mM})$ that the solution changed color (Chen et al., 2008). This behavior was explained in terms of the stiff $i$-motif structure that could not wrap around and stabilize the AuNPs, leading to their aggregation in the high ionic strength medium. The midpoint of the transition was around 6.8, slightly higher than the values obtained in the absence of AuNPs ( 6.5), which suggested a positive effect of AuNPs on the stability of $i$-motif DNA.

Most of the potential analytical methods dealing with $i$-motif structures (either as analyte or as reagent) are employed in small environments, such as cell metabolism; however, "canonical" spectroscopic or potentiometric methods frequently show a lack of spatial resolution when applied in a microscopic environment. Thus, a nanoscale $\mathrm{pH}$ probe was recently developed based on the above-mentioned interaction between $i$-motif and AuNPs (Zhao et al., 2013). The main novelty of this study was the use of morpholino oligomers (MO), nucleic acid analogs in which the sugar phosphate backbone of natural nucleic acid has been replaced by uncharged morpholine rings linked through phosphorodiamidate groups. This structure endowed morpholino oligomers with a better base stacking capacity than in natural DNA and greater water solubility, and the introduction of these oligomers into the assembly improved the stability of the $\mathrm{pH}$ probe even under low salt concentration. Due to the intense optical signal of AuNPs, local pH could be read out not only on the micro/nanofluidic channel but also on a single $i$-motif-MO-AuNP assembly. The $\mathrm{pH}$ probe showed a reversible and highly sensitive response to $\mathrm{pH}$ variation between 4.5 and 7.5.

Proper labeling of a cytosine-rich sequence with a fluorescent molecule may constitute a way to construct a pHsensitive sensor. In a recent work, the substitution of one of cytosines in three different internal positions of a sequence corresponding to the RET gene with its fluorescent analogue, 1,3-diaza-2-oxophenothiazine (tC), was reported (Bielecka and Juskowiak, 2015). This is a different approach to those previously described, in which dyes 
are attached to one or both ends of the cytosine-rich sequence. The $\mathrm{pH}$-induced i-motif formation resulted in fluorescence quenching of tC fluorophore. Even though fluorescence changes were reversible, $i$-motif folding $\mathrm{pH} 5.5$ needed a longer equilibration time comparing to unfolding process, which occurred at $\mathrm{pH}$ 7.5. Both folding and unfolding processes should be faster at the $\mathrm{pH}$ values tested in that work because of the intramolecular nature of folding. Hence, the slow fluorescence changes observed were related to the tC protonation. As the method exhibited linear response within $\mathrm{pH} 6.0$ and 7.0 with a resolution value below $0.1 \mathrm{pH}$, it was concluded that it could be used to monitor pH efficiently in biological systems. However, it is still under investigation how modified cytosine-rich sequences behave in presence of the complementary guanine-rich sequences. In a concomitant work, a fluorescent nucleoside analog composed of dimethylaniline fused to deoxycytidine was designed and synthesized (Mata and Luedtke, 2015). Interestingly, the modified nucleoside has the same pKa value ( $\sim .5)$ and base pairing characteristics as cytosine residues in $i$-motif structures. The results showed that the modified $i$-motif structure may pose large kinetic barriers to Watson-Crick duplex formation at $\mathrm{pH} 5.8,25^{\circ} \mathrm{C}$ and $100 \mathrm{mM} \mathrm{NaCl}$. This nucleoside analog could be used to develop further analytical methods.

Recently, a pH-sensitive system has been described that combines $i$-motif properties with the fluorescence signal of two pyrene molecules attached to both ends of a cytosine-rich oligonucleotide (Dembska and Juskowiak, 2015). In slightly acidic or even neutral $\mathrm{pH}$, the cytosine-rich part of the oligonucleotide probe folded into an $i$-motif structure, and an excimer (a complex created by the two pyrene molecules, one in an excited state and the other in the ground state) was formed. The characteristic feature of excimer emission was a long-wave fluorescence (maximum at $\sim 480 \mathrm{~nm}$ ) and a relatively long lifetime (30-60 ns) compared with the autofluorescence of cellular extracts (7 ns) (Kolpashchikov, 2010). Therefore, pyrene excimer fluorescence has been shown to be an excellent tool for bioanalytical applications when using time-resolved emission spectroscopy techniques. In contrast, when $\mathrm{pH}$ increased, the $i$-motif unfolded, causing the pyrene labels to move apart and shifting emission to monomer fluorescence (maximum at $\sim 400 \mathrm{~nm}$ ). The sensor gave an analytical response in excimer-monomer switching mode in a narrow $\mathrm{pH}$ range (1.5 $\mathrm{pH}$ units) and exhibited a relatively high $\mathrm{pH}$ resolution ( $0.1 \mathrm{pH}$ unit). Lastly, a fluorescent label-free pH sensor has been described based on an aggregation caused quenching (ACQ) probe (Fu et al., 2015). The label was a perylene tetracarboxylic acid diimide (PTDCI) derivative, a compound that has a propensity to form self-assembled linear chain structures and aggregates. When PTCDI monomers were arranged face-to-face, forming the above mentioned $\mathrm{H}$-aggregates, efficient fluorescence quenching occurred. Folding of cytosine-rich sequences 
into an i-motif structure dramatically affected the aggregation of PTCDI derivatives, releasing the monomers and providing significant fluorescence signals corresponding to the different $\mathrm{pH}$ value. The method was sensitive and provided reversible response to $\mathrm{pH}$ changes.

\subsection{Electrochemistry-based $\mathrm{pH}$ analysis}

Similarly to covalently attached dyes, cytosine-rich sequences may be modified at one or both ends with appropriate functional groups that allow the development of electrochemical methods for $\mathrm{pH}$ analysis.

A rather simple $i$-motif-based electrochemical pH sensor was devised by attaching a ferrocene-labeled cytosine-rich sequence onto a gold electrode (Xu et al., 2010). As with the AuNPs described above, the DNA sequence contained a thiol group at the $5^{\prime}$-end, enabling DNA to bind to the gold electrode surface. Variations in $\mathrm{pH}$ modified the distance between ferrocene moiety and electrode surface, leading to variation in the redox current. The $\mathrm{pH}$ could then be determined by measuring the corresponding currents. In the range of $\mathrm{pH}$ 5.6-7.1, a linear relationship was observed between the currents and $\mathrm{pH}$ values. The $\mathrm{pH}$ sensor also exhibited good selectivity with several common cations such as $\mathrm{Li}^{+}, \mathrm{Na}^{+}$or $\mathrm{K}^{+}$. In an attempt to reduce the background current, this set-up was later improved by using a system involving two cytosine-rich strands (Gao et al., 2012). As with Xu's method, the first strand was linked to the Au surface through a thiol group at the $5^{\prime}$ end, whereas the second strand contained a ferrocene group at the $5^{\prime}$ end

(Figure 4). At neutral and basic $\mathrm{pH}$, both strands hybridized with large bulges due to the presence of C.C mismatches. Under these conditions, the structure took the form of an extended Watson-Crick duplex that distanced ferrocene from the Au surface and in consequence, no intensity was observed. At slightly acidic $\mathrm{pH}$, the protonation of cytosine bases produced the formation of an intermolecular $i$-motif structure (involving both strands) that brought ferrocene closer to the electrode surface, producing a signal. The current had a linear relationship with $\mathrm{pH}$ values in the range of 5.8-8.0. The analytical signal obtained presented a much smaller background current than the previous intramolecular $i$-motif-based sensor, an improvement related to the extended duplex structure employed. The spatially separated configuration prevented electrical contact with the electrode, whereas the single-strand absorption at the electrode surface employed in the previous study could change the structure of the electrical double layer.

Electrodes based on boron-doped diamond (BDD) films present several interesting features such as high chemical stability, low detection limit, wide potential window and low background current. However, the range of functional 
groups that are required to immobilize biomolecules in these electrodes does not include the commonly used thiol groups. Hence, the use of a BDD electrode has been proposed that integrates AuNPs to permit efficient attachment of the ferrocene-tagged cytosine-rich DNA strand to the electrode surface (Song et al., 2012). As with previous methods, the distance between the labeled redox moiety and the electrode changed with $\mathrm{pH}$ value because of the formation of an $i$-motif structure. Sensor performance was improved due to the synergetic effects of the BDD electrode and the AuNPs, and the AuNP/BDD electrode exhibited higher sensitivity, a faster response, a wider linear range and good reproducibility for $\mathrm{pH}$ analysis compared with the planar Au electrode.

Recently, another application of thiol-modified cytosine-rich sequences has been reported. Here, crystal violet dye was used as a selective electrochemical probe for the $i$-motif structure because of its capacity to bind to the $i$-motif structure (Zhang et al., 2014). In acidic aqueous solution, crystal violet approached the electrode surface owing to the formation of the $i$-motif structure, resulting in a strong signal. In contrast, in neutral or basic aqueous solution, the $i$-motif structure unfolded, releasing crystal violet and yielding a weak signal. The measured current and pH showed a good linear relationship $(\mathrm{R}=0.989)$ in the range of $\mathrm{pH}$ 4.6-7.3. This $\mathrm{pH}$-driven electrochemical switch showed good reversibility and high selectivity over cations such as $\mathrm{Na}^{+}, \mathrm{K}^{+}$or $\mathrm{Mg}^{2+}$. The main disadvantage was the rather low stability constant of the dye-i-motif complex $\left(\sim 1.2 \cdot 10^{-6} \mathrm{M}^{-1}\right)$, obliging the use of relatively concentrated crystal violet solutions (up to $1 \mathrm{mM}$ ). The use of ligands showing stronger interaction with the $i$-motif could improve the performance of this electrode set-up.

\section{3. in vivo $\mathrm{pH}$ analysis}

It is important to measure $\mathrm{pH}$ in intracellular media because it plays critical roles in cellular activities such as proliferation, apoptosis, multidrug resistance, ion transport, endocytosis and muscle contraction (Huang et al., 2014). Abnormal pH can affect cellular internalization pathways and even the nervous system, causing diseases such as myocardial ischemia (Garlick et al., 1979) or cancer (Izumi et al., 2003).

Nucleic acid-based probes are not widely reported in live cell analysis because a live cell has a complex environment in which oligonucleotides can become unstable, or even degraded, due to interactions with other biomolecules.

Furthermore, it is difficult to affect the cellular uptake of the probes without the help of additional agents (Huang et al., 2014). As a result, there have been few reports to date on the use of $i$-motif-forming oligonucleotides for $\mathrm{pH}$ analysis in vivo. One of the first such applications was developed by Modi et al., who constructed a pH sensor based 
on the FRET mechanism (Modi et al., 2009). The sensor consisted of three oligonucleotides, 01,02 and 03 , where $\mathrm{O} 1$ and $\mathrm{O} 2$ were hybridized onto sites adjacent to $\mathrm{O3}$, leaving a one-base gap (Figure 5). $\mathrm{O} 1$ and $\mathrm{O} 2$ had singlestranded cytosine-rich overhangs designed in such a way that each overhang formed one-half of a bimolecular $i$ motif. At acidic $\mathrm{pH}$, these overhangs were protonated and the assembly folded to form an intermolecular $i$-motif. The $\mathrm{pH}$ sensor, fluorescently labeled at its $3^{\prime}$ and $5^{\prime}$ ends with Alexa-488 and Alexa-647 on 01 and 02 respectively, showed FRET at pH 5, with a transfer efficiency of 54-60\%. The method showed a dynamic range between $\mathrm{pH} 5.5$ and 6.8. To demonstrate its capacity to function inside living cells, the probe was used to map spatial and temporal $\mathrm{pH}$ changes associated with endosome maturation. Also, this sensor scheme was used to map pH changes associated with endocytosis in wild type as well as mutant worms of nematode Caenorhabditis elegans, demonstrating autonomous function within the organismal milieu in a variety of genetic backgrounds (Surana et al., 2011). Later, this $\mathrm{pH}$ sensor was improved by using two distinct DNA nanomachines that could be used simultaneously to map $\mathrm{pH}$ gradients along two different but intersecting cellular entry pathways (Modi et al., 2013). The two nanomachines, which were molecularly programmed to enter cells via different pathways, could map pH changes within welldefined subcellular environments along both pathways inside the same cell. These $\mathrm{pH}$ sensors were used to image $\mathrm{pH}$ values of early endosomes and the trans-Golgi network, in real time.

Another mechanism that has been used for the development of $\mathrm{pH}$ sensors is localized surface plasmon resonance (LSPR). One of the first applications of this mechanism was reported by Wang et al. The method was based on the formation of a core-satellite assembly, where the core was a 50nm AuNP carrying a guanine-rich sequence, and the satellite was a $14 \mathrm{~nm}$ AuNP carrying the complementary $i$-motif-forming cytosine-rich sequence (Wang et al., 2013). At neutral and basic $\mathrm{pH}$ values, the strands hybridized to form the Watson-Crick duplex. Under these conditions, LSPR of the assembly formed at a feeding core-satellite ratio of 1:200 exhibited a red-shift of $14 \mathrm{~nm}$ relative to that of the core AuNPs. At pH values near 5, the assembly could not be formed because the cytosine-rich sequence folded into an i-motif structure. In vivo, the core-satellite assemblies were absorbed into macrophage cells by endocytosis, allowing imaging of $\mathrm{pH}$ changes. Under the conditions reported in the study, within 30 min the yellow scattering signal from the core-satellite assembly in the cells became mostly green, suggesting its disassembly into elementary units inside acidic intracellular compartments. It is well-known that $\mathrm{pH}$ in the endocytic pathway drops from 5.9-6.2 in early endosomes to 4.7-5.5 in late endosomes/lysosomes, which agreed well with the observed imaging results. 
Recently, another intracellular probe has also been described, termed $i$-motif-based nanoflares (Huang et al., 2014). The nanoflares consisted of a $13 \mathrm{~nm}$ AuNP that was functionalized with 60 cytosine-rich sequences. These strands hybridized with fluorophore-labeled DNA molecules, termed "flares". Rhodamine Green was chosen as a fluorophore because its intensity is independent in the $\mathrm{pH}$ range $5.0-7.0$. In the bound state, the close proximity of the fluorophore to the AuNP surface led to fluorescence quenching. However, at acidic $\mathrm{pH}$, these cytosine-rich strands were protonated and folded to form an intramolecular $i$-motif. This folded structure disrupted Watson-Crick based-pairing between cytosine-rich strand and flare, causing flares to be liberated with an increase in fluorescence due to the greater distance of flares from the AuNP surface. Interestingly, the research included a systematic study of the influence of base mismatches on fluorescence at $\mathrm{pH} 7.0$ and $\mathrm{pH} 5.0$ and biological temperature $\left(37^{\circ} \mathrm{C}\right)$. The sequence that presented the maximum variation in fluorescence at this temperature was the one showing a mismatch. In vivo, analysis of intracellular nanoflare distribution suggested that the particles partly accumulated in the cell lysosome. The probe was also used to observe $\mathrm{pH}$ variation in cancer cells after treatment with chloroquine, a drug that may increase lysosome $\mathrm{pH}$.

A FRET-based probe has been developed consisting of a dual-fluorophore-labeled cytosine-rich sequence and its guanine-rich complementary strand linked to a gold nanoparticle (AuNP) (Huang et al., 2015b). At neutral pH, the FRET signal was low because of the separation of the two fluorophores due to the formation of the Watson-Crick duplex. At acidic $\mathrm{pH}$, the first strand folded into an intramolecular $i$-motif structure, with both $5^{\prime}$ and $3^{\prime}$ ends in close proximity, resulting in a high FRET efficiency. The fluorescence emission donor-to-acceptor ratio was used as a signal to measure $\mathrm{pH}$ in living cells. In this method, AuNPs were used as carriers because of their capacity for cellular transfection and enzymatic protection, as well as their low toxicity. The experimental results suggested that the $\mathrm{pH}$ sensor showed excellent spatial and temporal resolution in living cells.

The advantages of fluorescence imaging-based methods include high specificity and sensitivity. However, they also present some drawbacks, such as photobleaching, relatively high background noise and instability. In an attempt to overcome these problems, a pH-sensitive probe has been proposed based on DNA-modified AuNPs for Raman/fluorescence dual-imaging of intracellular pH distribution (Cao et al., 2015a). Raman spectroscopy can avoid photobleaching due to the extremely short lifetimes of Raman scattering; however, specificity is not as good as that of the fluorescent probes. By combining fluorescence and Raman spectroscopies, this set-up combined the advantages of both techniques. AuNPs were functionalized with a cytosine-rich strand with a thiol-moiety at the 3'- 
end for connection to the AuNP surface. The $5^{\prime}$-end of this sequence was labeled with a Raman signaling molecule, BHQ-2. At neutral pH values, the cytosine-rich strand was hybridized to its complementary guanine-rich strand, which was labeled at the fluorophore $\mathrm{Cy} 5$ at the $5^{\prime}$-end. At this $\mathrm{pH}$ value, the Watson-Crick double helix produced fluorescence quenching of $\mathrm{Cy} 5$ by both AuNP and $\mathrm{BHQ}-2$. In acidic $\mathrm{pH}$, the cytosine-rich strand folded into an intramolecular $i$-motif structure, releasing the complementary sequence. With the formation of the $i$-motif, the BHQ2 molecules were pulled closer to the surface of AuNPs, which enhanced the Raman response. At the same time, the Cy5-tagged complementary strand was released away from the AuNPs, which activated the fluorescence signal. Taking human cervical HeLa cells as a model, strong Raman and fluorescence signals were observed after endocytosis of the AuNP probe due to the acidic intracellular environment in which the in situ Raman/fluorescence dual-imaging of $\mathrm{pH}$ distribution in single cells had been performed. Compared with previously developed methods, the proposed probe only required a one-step incubation process, providing a $\mathrm{pH}$-sensitive dual-imaging strategy with a low background.

\subsection{Glucose and pyruvic acid analysis}

The $\mathrm{pH}$-driven conformational change in cytosine-rich sequences can also be used to indirectly determine analytes other than $\mathrm{H}_{3} \mathrm{O}^{+}$, such as glucose or pyruvic acid. Recently, a electrochemical sensor has been developed to analyze glucose and urea (Gao et al., 2015). The addition of glucose or urea activated a glucose oxidase-catalyzed or ureasecatalyzed reaction, inducing or destroying the formation of the $i$-motif structure. The conformational switch of the oligonucleotide probe was recorded by electrochemical impedance spectroscopy. Thus, the difference in electron transfer resistance was utilized to quantitatively determine glucose and urea. The sensor exhibited high selectivity, excellent stability and a remarkable capacity for regeneration.

A fluorescence biosensor for detecting glucose has been proposed based on the $\mathrm{pH}$-induced conformational switch of $i$-motif DNA (Ke et al., 2014). Glucose oxidized by molecular oxygen in the presence of glucose oxidase (GOD) produced gluconic acid and decreased the $\mathrm{pH}$ value of the medium. This induced the fluorophore- and quencherlabeled cytosine-rich DNA to fold into the $i$-motif structure. As a result, fluorescence quenching occurred because of the resonance energy transfer between fluorophore and quencher. Based on this working principle, the concentration of glucose was quantified by measuring the decrease in fluorescence signal. Under optimal experimental conditions, the assay showed a linear response range of $-100 \mu \mathrm{M}$ for the glucose concentration with a 
detection limit of $4 \mu \mathrm{M}$. This glucose biosensor was successfully applied to determine glucose in real samples, suggesting its potential as a practical application.

Besides $\mathrm{pH}$, several methods using AuNP technology have also been developed to analyze glucose. As with the previously mentioned method, Li et al. developed a selective and sensitive glucose sensor based on measuring the $\mathrm{H}_{3} \mathrm{O}^{+}$concentration due to the presence of gluconic acid (Li et al., 2012). In the absence of glucose, cytosine-rich strands unfolded, stabilizing and protecting the AuNPs. By adding glucose oxidase to a sample containing glucose, gluconic acid was generated and consequently, the $\mathrm{pH}$ value decreased. Under these conditions, formation of the $i$ motif structure hindered stabilization of the AuNPs. The change in color of AuNPs from red to blue was easily detected by eye or with a molecular absorption spectrophotometer. Also based on the use of AuNPs, a selective spectroscopic method has been proposed for detecting pyruvic acid (Li et al., 2014). In the presence of pyruvate decarboxylase, pyruvic acid was quantitatively converted to acetaldehyde and $\mathrm{CO}_{2}$, causing the solution $\mathrm{pH}$ to change from acidic to neutral. As in other studies, AuNPs were bonded to a single strand cytosine-rich sequence at neutral $\mathrm{pH}$ values. Upon decarboxylation, $\mathrm{pH}$ decreased and the $i$-motif structure was formed, thus releasing the AuNPs and affecting their optical properties. The absorbance difference before and after addition of decarboxylase depends on analyte concentration, and was used for its determination. The method yielded a linear range from 6 . $10^{-6} \mathrm{M}$ to $2 \cdot 10^{-4} \mathrm{M}$ and a detection limit of $3 \mu \mathrm{M}$.

\subsection{Other applications}

Other reported applications for chemical analysis include determination of diverse analytes, such as carbon nanotubes, $\mathrm{Ag}^{+}$ions or proteins.

Due to the increasing worldwide demand for carbon nanotubes and growing concerns about their safe development and use, analytical methods are required for their detection and quantitation. Thus, an analytical method has been described based on the capacity of single-walled carbon nanotubes (SWNTs) to specifically induce $i$-motif formation (Peng et al., 2009). The sensor consisted of a short single-stranded DNA containing the human telomeric sequence $5^{\prime}-\left(C_{3} T_{2}\right)_{4}-3^{\prime}$, as well as a linker sequence to ensure solvent exposure and an unfolded state at pH 7.0. This DNA, modified with redox-active methylene blue (MB) at its $3^{\prime}$-end, was covalently attached at its $5^{\prime}$-end to a gold electrode. At pH 7.0, the measured current was due to the attached MB tag, which came within close proximity of the electrode surface and collided with (or weakly bound to) the electrode and transferred electrons. SWNTs 
selectively induced $i$-motif formation of the immobilized human telomeric sequence on the surface. Hence, when the i-motif DNA-modified electrode was immersed in buffer ( $\mathrm{pH}$ 7.0) containing $5 \mathrm{ppm}$ SWNTs for 1 hour at room temperature, the current decreased dramatically, suggesting that the MB tag was held far from the electrode surface upon SWNT binding. The effect was explained in terms of stabilization of the $i$-motif structure by SWNT at pH 7.0. The method, which was applied to SWNT analysis in cell extracts, had a detection limit of $0.2 \mathrm{ppm}$.

Cytosine-rich sequences have also been used to detect inorganic ions, such as $\mathrm{Ag}^{+}$. For example, a sensor has been designed based on the interaction of a cytosine-rich strand with $\mathrm{Ag}^{+}$, which was sensitively recognized by the 2,2diethyl-9-methyl-selenacarbocyanine bromide (DMSB) fluorescent dye (Shi et al., 2015). Upon interaction, the fluorescence intensity of DMSB was enhanced dramatically, eventually enabling the quantitation of $\mathrm{Ag}^{+}$. Recently, a fluorescence-based method has been proposed consisting of DNA-templated silver nanoclusters (DNA-AgNCs) (Lee et al., 2015b). $\mathrm{Ag}^{+}$ions induced a dimeric structure of $\mathrm{C}_{12}-\mathrm{AgNCs}$ by forming a bridge between two $\mathrm{C}_{12}-\mathrm{AgNCs}$, where $\mathrm{C}_{12}$ was a DNA strand consisting of 12 cytosines bases. In a similar way to the previously mentioned optical properties of AuNPs, the dimer formation caused the $\mathrm{C}_{12}$-AgNC fluorescence to change from red to green. Using this $\mathrm{Ag}^{+}$-triggered fluorescence switch, $\mathrm{Ag}^{+}$was detected at concentrations as low as $10 \mathrm{nM}$.

Lastly, the use has been reported of cytosine-rich DNA sequences to quantify the activity of terminal deoxynucleotidyl transferase (TdT) (Lu et al., 2015). TdT is a template-free polymerase that catalyzes the random addition of deoxyribonucleoside triphosphates (dNTP) to the $3^{\prime}-\mathrm{OH}$ terminus of single-stranded DNA and bluntended or 3'-protruding double-stranded DNA fragments. Hence, TdT generates DNA sequences that are largely dependent on the composition of the substrate dNTP pool. Analysis of this protein is of interest not only because TdT is widely used as a tool to detect DNA, RNA, protein and DNA-modifying enzymes, but also because of its value as a biomarker for acute leukemia. The proposed analytical method for TdT quantitation was based on a cyclometalated iridium(III) complex that exhibited specific interaction with $i$-motif structures over a Watson-Crick duplex or single strand. The mechanism of the proposed method was as follows: the DNA primer (5'GTTAACCTAGCCAG-3') was incubated with TdT in the presence of a C-rich dNTP substrate pool. The nascent cytosine-rich sequence folded into the $i$-motif structure under acidic conditions, which could then be recognized by the luminescent iridium(III) complex with an enhanced luminescence response. The method exhibited a linear range of detection in the concentration range of 0 to $8 \mathrm{U} \cdot \mathrm{mL}^{-1}$, and the limit of detection for TdT was $0.25 \mathrm{U} \cdot \mathrm{mL}^{-1}$ (Lu et al., 2015). The selectivity of the $i$-motif-based TdT activity detection platform was tested by investigating the response of 
the assay to four kinds of polymerases and six other DNA-modifying enzymes. The results indicated that the system displayed superior selectivity to TdT over other DNA-modifying enzymes, which presumably originated from the specific generation of C-rich DNA by TdT in the detection system.

\section{Perspectives and conclusions}

This paper provides an overview of the main applications of $i$-motif structures in chemical analysis. $i$-motif formation and stability are strongly dependent on $\mathrm{pH}$; therefore, all the analytical methods reviewed here are related to $\mathrm{pH}$. The main advantages conferred by this structure are its binary, fast and reversible response in the $\mathrm{pH}$ range of approximately 5-8. Moreover, analytical features such as sensitivity or linear range may be modified by appropriate tuning of cytosine-rich sequences, for example by changing the number and / or nature of bases at the loops, or the length of cytosine segments. Chemical modifications at appropriate positions, such as the $5^{\prime}$ or $3^{\prime}$ ends, with optically- and / or electrochemically-active ligands allows the application of diverse instrumental techniques. The most recent applications describe nucleic acids attached to surfaces, facilitating analysis at single-molecule level in nanoscale environments. On the other hand, the development of analytical methods based on the folding events of this structure may be hindered because of the presence of some difficulties. First, the $\mathrm{pH}$-induced transition takes place at $\mathrm{pH}$ values around 6-7, approximately, at room temperature, which makes difficult the measurement at basic $\mathrm{pH}$ values. Besides the fact that work is currently done to increase the stability of $i$-motif structures at neutral and slightly basic $\mathrm{pH}$ values, it is not clear that this structure could be used to map more basic $\mathrm{pH}$ values. Second, the application in vivo of these structures must take into account the presence of biological interferences, being probably the most evident the presence of guanine-rich sequences. It has been demonstrated that at slightly acid and neutral $\mathrm{pH}$ values, the formation of Watson-Crick duplex overrules that of $i$-motif structures.

In perspective, use of backbone-modified $i$-motif-forming sequences is still a matter of research. It has been shown that such modifications affect the stability of these structures, which in turn could be used to modify their analytical properties. In addition, current research on the potential role in vivo of these structures may reveal new targets for analytical methods, such as specific proteins, which could be quantified in vivo. 


\section{Acknowledgments}

We thank the Spanish government (CTQ2012-38616-C02-02 and CTQ2014-52588-R) for funding and the Catalan government (2014 SGR 1106) for recognition. 


\section{Figures}

Figure 1. (A) Scheme of the C.C. $C^{+}$base pair and of an intramolecular i-motif structure. Six $C \cdot C^{+}$base pairs are depicted. Bases other than cytosines, which are not involved in the formation of the $i$-motif core, have been omitted. (B) Distribution diagram showing the $\mathrm{pH}$ range of existence for the $5^{\prime}-\left(\mathrm{C}_{3} \mathrm{~T}_{3}\right)_{4}-3^{\prime}$ and the strong cooperative effects that accompany its formation (Fernandez et al., 2011).

Figure 2. Several ligands used for detection and analytical applications of the $i$-motif structure: (A) Crystal Violet, (B) Thiazol Orange, (C) Thioflavin, (D) Polythiophene, (E) Morpholine, (F) Ferrocene

Figure 3. (A) A Schematic illustration of the working cycle and color change of AuNP-DNA assembly at different $\mathrm{pH}$ of the solution and DNA sequences used herein, where NP1 indicates the AuNP modified with i-motif DNA and NP2 is NP modified with the complementary guanine-rich DNA. The sequences of the DNA on each AuNPs are written out at the bottom of the scheme. (B) A picture showing the reversibility and color change of the DNA-gold conjugates at different $\mathrm{pH}$. The pictures are taken $30 \mathrm{~min}$ after each $\mathrm{pH}$ change. (Sharma et al., 2007). Reprinted with permission.

Figure 4. Scheme of an electrochemistry-based $\mathrm{pH}$ sensor. The signal is produced when ferrocene $(\mathrm{Fc})$ tag is close to the gold electrode surface. In the "OFF" state (left), a Watson-Crick duplex is formed and Fc is far from the electrode surface. On the other hand, in the "ON" state (right), cytosine bases in mismatches form an intramolecular i-motif structure, allowing the approach of Fc to the electrode surface. (Gao et al., 2012) Reprinted with permission.

Figure 5. Scheme of a FRET-based sensor consisting of three oligonucleotides, O1, O2 and O3 (Modi et al., 2009). Upon addition of $\mathrm{H}^{+}$, a bimolecular i-motif structure is formed by oligonucleotides $\mathrm{O} 1$ and $\mathrm{O} 2$. Note that this is one of the few examples of bimolecular i-motifs used in the development of analytical methods. 


\section{Bibliography}

Amato, J., laccarino, N., Randazzo, A., Novellino, E., and Pagano, B., Noncanonical DNA Secondary Structures as Drug Targets: the Prospect of the i-Motif. ChemMedChem, 2014, 9, 2026-2030.

Benabou, S., Avino, A., Eritja, R., Gonzalez, C., and Gargallo, R., Fundamental aspects of the nucleic acid i-motif structures. RSC Adv., 2014, 4, 26956-26980.

Bielecka, P. and Juskowiak, B., Fluorescent Sensor for pH Monitoring Based on an i-Motif - - Switching Aptamer Containing a Tricyclic Cytosine Analogue (tC). Molecules, 2015, 20, 18511-18525.

Brazier, J. A., Shah, A., and Brown, G. D., I-Motif formation in gene promoters: unusually stable formation in sequences complementary to known G-quadruplexes. Chem. Commun., 2012, 48, 10739-10741.

Brooks, T. A., Kendrick, S., and Hurley, L., Making sense of G-quadruplex and i-motif functions in oncogene promoters. FEBS Journal, 2010, 277, 3459-3469.

Bucek, P., Gargallo, R., and Kudrev, A., Spectrometric study of the folding process of i-motif-forming DNA sequences upstream of the c-kit transcription initiation site. Anal. Chim. Acta, 2010, 683, 69-77.

Canalia, M. and Leroy, J. L., Structure, internal motions and association-dissociation kinetics of the i-motif dimer of d(5mCCTCACTCC). Nucleic Acids Res., 2005, 33, 5471-5481.

Canalia, M. and Leroy, J. L., [5mCCTCTCTCC]4: An i-motif tetramer with intercalated T·T pairs. J. Am. Chem. Soc., 2009, 131, 12870-12871.

Cao, Y., Qian, R.-C., Li, D.-W., and Long, Y.-T., Raman/fluorescence dual-sensing and imaging of intracellular pH distribution. Chem. Commun., 2015a, 51, 17584-17587.

Cao, Y., Qin, Y., Bruist, M., Gao, S., Wang, B., Wang, H., and Guo, X., Formation and Dissociation of the Interstrand iMotif by the Sequences $\mathrm{d}(\mathrm{XnC} 4 \mathrm{Ym})$ Monitored with Electrospray lonization Mass Spectrometry. J. Am. Soc. Mass. Spectrom., 2015b, 26, 994-1003.

Collin, D. and Gehring, K., Stability of chimeric DNA/RNA cytosine tetrads: Implications for i-motif formation by RNA. J. Am. Chem. Soc., 1998, 120, 4069-4072.

Chen, C., Song, G., Ren, J., and Qu, X., A simple and sensitive colorimetric $\mathrm{pH}$ meter based on DNA conformational switch and gold nanoparticle aggregation. Chem. Commun., 2008, 46, 6149-6151.

Day, H. A., Pavlou, P., and Waller, Z. A. E., i-Motif DNA: Structure, stability and targeting with ligands. Biorg. Med. Chem., 2014, 22, 4407-4418.

Dembska, A. and Juskowiak, B., Pyrene functionalized molecular beacon with $\mathrm{pH}$-sensitive i-motif in a loop. Spectrochimica Acta Part A: Molecular and Biomolecular Spectroscopy, 2015, 150, 928-933.

Dexheimer, T. S., Carey, S. S., Zuohe, S., Gokhale, V. M., Hu, X., Murata, L. B., Maes, E. M., Weichsel, A., Sun, D., Meuillet, E. J., Montfort, W. R., and Hurley, L. H., NM23-H2 may play an indirect role in transcriptional activation of c-myc gene expression but does not cleave the nuclease hypersensitive element III 1. Molecular Cancer Therapeutics, 2009, 8, 1363-1377.

Dong, Y., Yang, Z., and Liu, D., DNA nanotechnology based on i-motif structures. Acc. Chem. Res., 2014, 47, 18531860.

Fenn, S., Du, Z., Lee, J. K., Tjhen, R., Stroud, R. M., and James, T. L., Crystal structure of the third KH domain of human poly $(\mathrm{C})$-binding protein-2 in complex with a C-rich strand of human telomeric DNA at 1.6 A resolution. Nucleic Acids Res., 2007, 35, 2651-2660.

Fernandez, S., Eritja, R., Aviño, A., Jaumot, J., and Gargallo, R., Influence of pH, temperature and the cationic porphyrin TMPyP4 on the stability of the i-motif formed by the 5'-(C3TA2)4-3' sequence of the human telomere. Int. J. Biol. Macromol., 2011, 49, 729-736.

Fu, B., Huang, J., Bai, D., Xie, Y., Wang, Y., Wang, S., and Zhou, X., Label-free detection of pH based on the i-motif using an aggregation-caused quenching strategy. Chem. Commun., 2015, 51, 16960-16963 
Fujii, T. and Sugimoto, N., Loop nucleotides impact the stability of intrastrand i-motif structures at neutral pH. PCCP, 2015, 17, 16719-16722.

Gallego, J., Chou, S. H., and Reid, B. R., Centromeric pyrimidine strands fold into an intercalated motif by forming a double hairpin with a novel T:G:G:T tetrad: Solution structure of the d(TCCCGTTTCCA) dimer. J. Mol. Biol., $1997,273,840-856$.

Gao, X., Li, X., Xiong, W., Huang, H., Lin, Z., Qiu, B., and Chen, G., I-Motif based pH induced electrochemical switches. Electrochem. Commun., 2012, 24, 9-12.

Gao, Z. F., Chen, D. M., Lei, J. L., Luo, H. Q., and Li, N. B., A regenerated electrochemical biosensor for label-free detection of glucose and urea based on conformational switch of i-motif oligonucleotide probe. Anal. Chim. Acta, 2015, 897, 10-16.

Garlick, P. B., Radda, G. K., and Seeley, P. J., Studies of acidosis in the ischaemic heart by phosphorus nuclear magnetic resonance. Biochem. J, 1979, 184, 547-554.

Gurung, S. P., Schwarz, C., Hall, J. P., Cardin, C. J., and Brazier, J. A., The importance of loop length on the stability of i-motif structures. Chem. Commun., 2015, 51, 5630-5632.

Huang, H., Hong, X., Liu, F., and Li, N., A simple approach to study the conformational switching of i-motif DNA by fluorescence anisotropy. Analyst, 2015a, 140, 5987-5991.

Huang, J., He, Y., Yang, X., Wang, K., Ying, L., Quan, K., Yang, Y., and Yin, B., I-motif-based nano-flares for sensing pH changes in live cells. Chem. Commun., 2014, 50, 15768-15771.

Huang, J., Ying, L., Yang, X., Yang, Y., Quan, K., Wang, H., Xie, N., Ou, M., Zhou, Q., and Wang, K., Ratiometric Fluorescent Sensing of pH Values in Living Cells by Dual-Fluorophore-Labeled i-Motif Nanoprobes. Anal. Chem., 2015b, 87, 8724-8731.

Izumi, H., Torigoe, T., Ishiguchi, H., Uramoto, H., Yoshida, Y., Tanabe, M., Ise, T., Murakami, T., Yoshida, T., Nomoto, M., and Kohno, K., Cellular pH regulators: potentially promising molecular targets for cancer chemotherapy. Cancer Treatment Reviews, 2003, 29, 541-549.

Ke, Q., Zheng, Y., Yang, F., Zhang, H., and Yang, X., A fluorescence glucose sensor based on pH induced conformational switch of i-motif DNA. Talanta, 2014, 129, 539-544.

Kendrick, S., Akiyama, Y., Hecht, S. M., and Hurley, L. H., The i-motif in the bcl-2 P1 promoter forms an unexpectedly stable structure with a unique 8:5:7 loop folding pattern. J. Am. Chem. Soc., 2009, 131, 17667 - 17676.

Kendrick, S., Kang, H.-J., Alam, M. P., Madathil, M. M., Agrawal, P., Gokhale, V., Yang, D., Hecht, S. M., and Hurley, L. H., The Dynamic Character of the BCL2 Promoter i-Motif Provides a Mechanism for Modulation of Gene Expression by Compounds That Bind Selectively to the Alternative DNA Hairpin Structure. J. Am. Chem. Soc., 2014, 136, 4161-4171.

Khan, N., Aviño, A., Tauler, R., Gonzalez, C., Eritja, R., and Gargallo, R., Solution equilibria of the i-motif-forming region upstream of the B-cell lymphoma-2 P1 promoter. Biochimie, 2007, 89, 1562-1572.

Kolpashchikov, D. M., Binary probes for nucleic acid analysis. Chem. Rev., 2010, 110, 4709-4723.

Kumar, N., Petersen, M., and Maiti, S., Tunable c-MYC LNA i-motif. Chem. Commun., 2009, 1532-1534.

Lacroix, L., Lienard, H., Labourier, E., Djavaheri-Mergny, M., Lacoste, J., Leffers, H., Tazi, J., Helene, C., and Mergny, J. L., Identification of two human nuclear proteins that recognise the cytosine-rich strand of human telomeres in vitro. Nucleic Acids Res., 2000, 28, 1564-1575.

Lacroix, L., Mergny, J. L., Leroy, J. L., and Helene, C., Inability of RNA to form the i-motif: Implications for triplex formation. Biochemistry, 1996, 35, 8715-8722.

Lannes, L., Halder, S., Krishnan, Y., and Schwalbe, H., Tuning the pH Response of i-Motif DNA Oligonucleotides. ChemBioChem, 2015, 16, 1647-1656.

Largy, E. and Mergny, J.-L., Shape matters: size-exclusion HPLC for the study of nucleic acid structural polymorphism. Nucleic Acids Res., 2014, 42, e149.

Lee, I. J., P. Patil, S., Fhayli, K., Alsaiari, S., and Khashab, N. M., Probing structural changes of self assembled i-motif DNA. Chem. Commun., 2015a, 51, 3747-3749. 
Lee, I. J., Park, M., Joo, T., and Kim, B. H., Using fluorescence changes of F1U units at terminal and mid-loop positions to probe i-motif structures. Molecular BioSystems, 2012, 8, 486-490.

Lee, I. J., Yi, J. W., and Kim, B. H., Probe for i-motif structure and G-rich strands using end-stacking ability. Chem. Commun., 2009, 5383-5385.

Lee, J., Park, J., Hee Lee, H., Park, H., Kim, H. I., and Kim, W. J., Fluorescence switch for silver ion detection utilizing dimerization of DNA-Ag nanoclusters. Biosens. Bioelectron., 2015b, 68, 642-647.

Leroy, J. L., The formation pathway of i-motif tetramers. Nucleic Acids Res., 2009, 37, 4127-4134.

Leroy, J. L., Gueron, M., Mergny, J. L., and Helene, C., Intramolecular Folding of a Fragment of the Cytosine-Rich Strand of Telomeric DNA into an I-Motif. Nucleic Acids Res., 1994, 22, 1600-1606.

Li, J., Huang, Y. Q., Qin, W. S., Liu, X. F., and Huang, W., An optical-logic system based on cationic conjugated polymer/DNA/ intercalating dyes assembly for label-free detection of conformational conversion of DNA imotif structure. Polymer Chemistry, 2011, 2, 1341-1346.

Li, W., Feng, L., Ren, J., Wu, L., and Qu, X., Visual detection of glucose using conformational switch of i-motif DNA and non-crosslinking gold nanoparticles. Chemistry - A European Journal, 2012, 18, 12637-12642.

Li, W., Pan, C., Hou, T., Wang, X., and Li, F., Selective and colorimetric detection of pyruvic acid using conformational switch of i-motif DNA and unmodified gold nanoparticles. Analytical Methods, 2014, 6, 1645-1649.

Lieblein, A. L., Fürtig, B., and Schwalbe, H., Optimizing the Kinetics and Thermodynamics of DNA i-Motif Folding. ChemBioChem, 2013, 14, 1226-1230.

Liu, D. and Balasubramanian, S., A Proton-Fuelled DNA Nanomachine. Angewandte Chemie - International Edition, 2003, 42, 5734-5736.

Loweth, C. J., Brett Caldwell, W., Peng, X., Alivisatos, A. P., and Schultz, P. G., DNA-based assembly of gold nanocrystals. Angewandte Chemie - International Edition, 1999, 38, 1808-1812.

Lu, L., Wang, M., Liu, L.-J., Wong, C.-Y., Leung, C.-H., and Ma, D.-L., A luminescence switch-on probe for terminal deoxynucleotidyl transferase (TdT) activity detection by using an iridium(iii)-based i-motif probe. Chem. Commun., 2015, 51, 9953-9956.

Ma, D. L., Kwan, M. H. T., Chan, D. S. H., Lee, P., Yang, H., Ma, V. P. Y., Bai, L. P., Jiang, Z. H., and Leung, C. H., Crystal violet as a fluorescent switch-on probe for i-motif: Label-free DNA-based logic gate. Analyst, 2011, 136, 2692-2696.

Mata, G. and Luedtke, N. W., A Fluorescent Probe for Proton-Coupled Folding Reveals Slow Exchange of i-Motif and Duplex DNA. J. Am. Chem. Soc., 2015, 137, 699-707.

Mergny, J. L., Lacroix, L., Han, X., Leroy, J. L., and Helene, C., Intramolecular folding of pyrimidine oligodeoxynucleotides into a i-DNA motif. J. Am. Chem. Soc., 1995, 117, 8887-8898.

Modi, S., Nizak, C., Surana, S., Halder, S., and Krishnan, Y., Two DNA nanomachines map pH changes along intersecting endocytic pathways inside the same cell. Nat Nano, 2013, 8, 459-467.

Modi, S., Swetha, M. G., Goswami, D., Gupta, G. D., Mayor, S., and Krishnan, Y., A DNA nanomachine that maps spatial and temporal pH changes inside living cells. Nat Nano, 2009, 4, 325-330.

Nesterova, I. V., Elsiddieg, S. O., and Nesterov, E. E., Design and evaluation of an i-motif-based allosteric control mechanism in DNA-hairpin molecular devices. J. Phys. Chem. B, 2013, 117, 10115-10121.

Nesterova, I. V. and Nesterov, E. E., Rational Design of Highly Responsive pH Sensors Based on DNA i-Motif. J. Am. Chem. Soc., 2014, 136, 8843-8846.

Peng, Y., Wang, X., Xiao, Y., Feng, L., Zhao, C., Ren, J., and Qu, X., i-Motif Quadruplex DNA-Based Biosensor for Distinguishing Single- and Multiwalled Carbon Nanotubes. J. Am. Chem. Soc., 2009, 131, 13813-13818.

Perez-Rentero, S., Gargallo, R., Gonzalez, C., and Eritja, R., Modulation of the stability of i-motif structures using an acyclic threoninol cytidine derivative. RSC Adv., 2015, 5, 63278-63281.

Reilly, S. M., Morgan, R. K., Brooks, T. A., and Wadkins, R. M., Effect of Interior Loop Length on the Thermal Stability and pKa of i-Motif DNA. Biochemistry, 2015, 54, 1364-1370. 
Ren, X., He, F., and Xu, Q. H., Direct visualization of conformational switch of i-motif DNA with a cationic conjugated polymer. Chemistry - An Asian Journal, 2010, 5, 1094-1098.

Rosu, F., Gabelica, V., Joly, L., Gregoire, G., and De Pauw, E., Zwitterionic i-motif structures are preserved in DNA negatively charged ions produced by electrospray mass spectrometry. PCCP, 2010, 12, 13448-13454.

Ruedas-Rama, M. J., Orte, A., Martin-Domingo, M. C., Castello, F., Talavera, E. M., and Alvarez-Pez, J. M., Interaction of YOYO-3 with different DNA templates to form H-aggregates. J. Phys. Chem. B, 2014, 118, 6098-6106.

Sharma, J., Chhabra, R., Yan, H., and Liu, Y., pH-driven conformational switch of "i-motif" DNA for the reversible assembly of gold nanoparticles. Chem. Commun., 2007, 477-479.

Shi, Y., Sun, H., Xiang, J., Yu, L., Yang, Q., Li, Q., Guan, A., and Tang, Y., i-Motif-modulated fluorescence detection of silver(I) with an ultrahigh specificity. Anal. Chim. Acta, 2015, 857, 79-84.

Simonsson, T., Pribylova, M., and Vorlickova, M., A nuclease hypersensitive element in the human c-myc promoter adopts several distinct i-tetraplex structures. Biochem. Biophys. Res. Commun., 2000, 278, 158-166.

Song, M. J., Lee, S. K., Lee, J. Y., Kim, J. H., and Lim, D. S., Electrochemical sensor based on Au nanoparticles decorated boron-doped diamond electrode using ferrocene-tagged aptamer for proton detection. $J$. Electroanal. Chem., 2012, 677-680, 139-144.

Surana, S., Bhat, J. M., Koushika, S. P., and Krishnan, Y., An autonomous DNA nanomachine maps spatiotemporal pH changes in a multicellular living organism. Nat Commun, 2011, 2, 340.

Teller, C. and Willner, I., Functional nucleic acid nanostructures and DNA machines. Curr. Opin. Biotechnol., 2010, 21, 376-391.

Wang, C., Du, Y., Wu, Q., Xuan, S., Zhou, J., Song, J., Shao, F., and Duan, H., Stimuli-responsive plasmonic coresatellite assemblies: I-motif DNA linker enabled intracellular pH sensing. Chem. Commun., 2013, 49, 57395741.

Wang, L., Liu, X., Yang, Q., Fan, Q., Song, S., Fan, C., and Huang, W., A colorimetric strategy based on a water-soluble conjugated polymer for sensing $\mathrm{pH}$-driven conformational conversion of DNA i-motif structure. Biosens. Bioelectron., 2010, 25, 1838-1842.

Webb, B. A., Chimenti, M., Jacobson, M. P., and Barber, D. L., Dysregulated pH: a perfect storm for cancer progression. Nat Rev Cancer, 2011, 11, 671-677.

Xu, B., Wu, X., Yeow, E. K. L., and Shao, F., A single thiazole orange molecule forms an exciplex in a DNA i-motif. Chem. Commun., 2014, 50, 6402-6405.

Xu, X., Li, B., Xie, X., Li, X., Shen, L., and Shao, Y., An i-DNA based electrochemical sensor for proton detection. Talanta, 2010, 82, 1122-1125.

Zhang, X. Y., Luo, H. Q., and Li, N. B., Crystal violet as an i-motif structure probe for reversible and label-free pHdriven electrochemical switch. Anal. Biochem., 2014, 455, 55-59.

Zhao, Y., Cao, L., Ouyang, J., Wang, M., Wang, K., and Xia, X. H., Reversible plasmonic probe sensitive for pH in micro/nanospaces based on i-motif-modulated morpholino-gold nanoparticle assembly. Anal. Chem., 2013, 85, 1053-1057.

Zhou, T., Chen, P., Niu, L., Jin, J., Liang, D., Li, Z., Yang, Z., and Liu, D., pH-responsive size-tunable self-assembled DNA dendrimers. Angewandte Chemie - International Edition, 2012, 51, 11271-11274. 